\section{STELLAR SHOW}

Scientists have struggled to explain the erratic behaviour of the binary star $n$ Carinae, which brightened unexpectedly in the 1840s and again more recently.

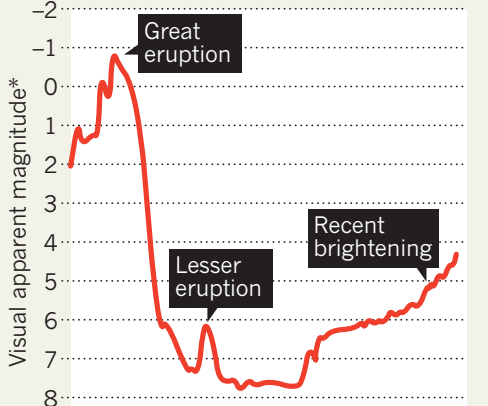

$\begin{array}{llllll}1820 & 1860 & 1900 & 1940 & 1980 & 2020\end{array}$ * Lower numbers represent greater brightness.

X-ray production peaked in mid-July and has since plummeted to near zero probably as the colliding winds, where the X-rays are born, have become entirely unstable and collapsed.

The Hubble Space Telescope and other instruments are also tracking dramatic changes in the chemical-element signatures found in $\eta$ Carinae's light spectrum. The interaction between the two approaching stars can strip electrons from elements such as iron and helium, ionizing them more strongly than in normal celestial environments. "You have these bare helium nuclei — that's awfully hard to make in normal circumstances," says Gull. Watching this process over time helps to reveal how the stellar winds are interacting.

At the Pico dos Dias Observatory in southern Brazil, astronomer Augusto Damineli has been spending every night since 25 July trying to catch a glimpse of $\eta$ Carinae through the winter clouds. On 29 July, his team finally caught a brief opening and managed to gather data showing that a helium spectral line is dropping in just the pattern that Damineli expected. "TOUCHDOWN!" he wrote in an e-mail.

In 2009, when $\eta$ Carinae had its most recent close encounter, the system's X-ray production plunged and then shot back up in half the time it did in 2003. That could be because the primary star's winds are slowing down, so it takes less time for the whole system to recover. If the wind speeds have continued to drop, X-ray emissions might shoot up even faster than last time.

Seeing such big differences from one close encounter to the next is "what everyone is waiting for", says Andrea Mehner, an astronomer at the European Southern Observatory in Santiago, Chile, who is monitoring $\eta$ Carinae with Hubble. "We cannot make the star do something exciting if it doesn't want to."

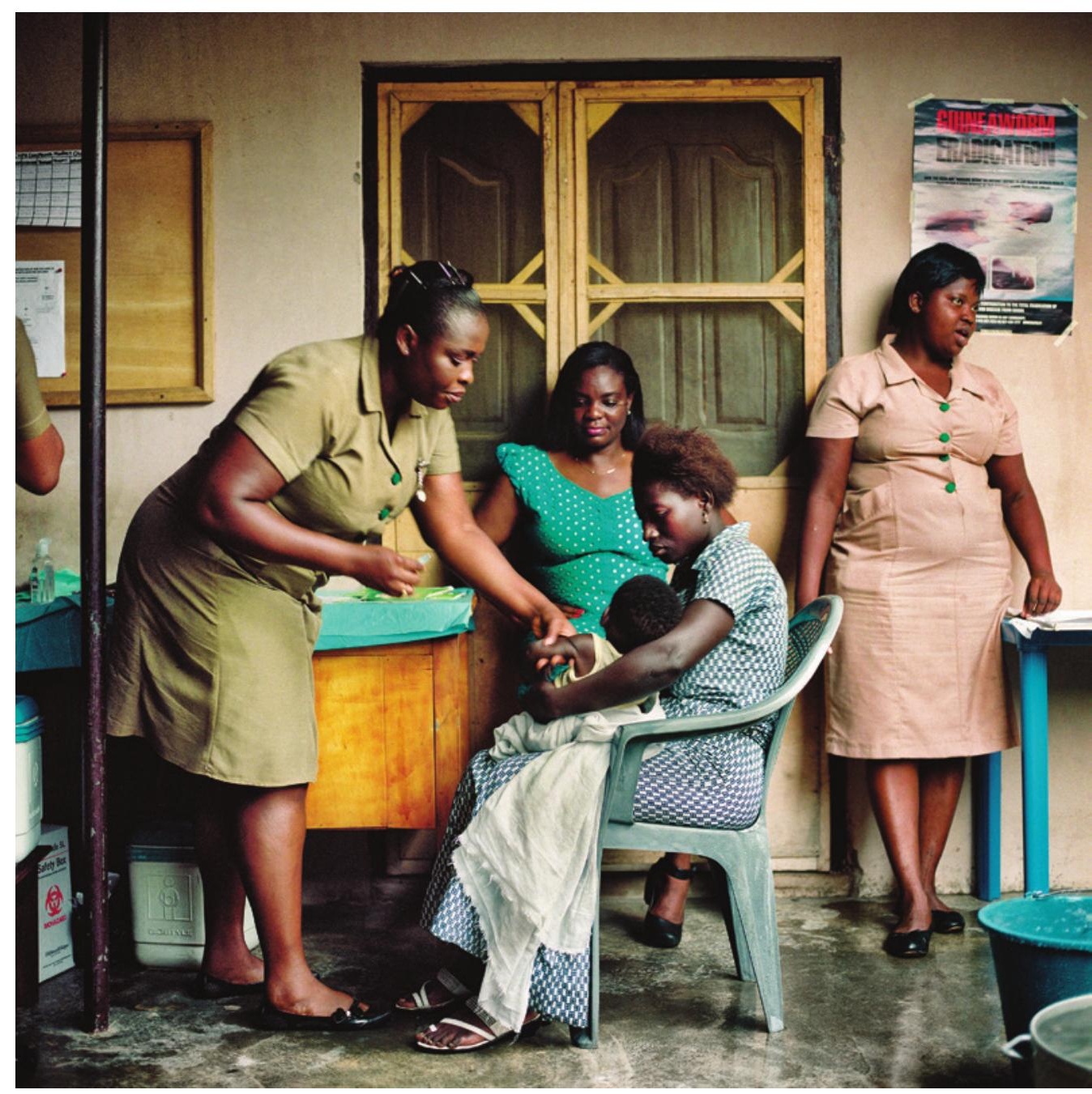

A nurse prepares to immunize a young child with pneumococcal and rotavirus vaccines in Ghana.

\title{
VACCINES
}

\section{Hidden bonus from vaccination}

\section{Immunization against pneumococcus in Africa also reduces levels of antibiotic resistance.}

\section{BY EWEN CALLAWAY}

$\mathrm{T}$

This summer, Eritrea, Côte d'Ivoire and Niger will join a growing list of coun-

tries where infants receive a vaccine to prevent pneumonia, meningitis and other deadly diseases caused by the pneumococcus bacterium (Streptococcus pneumoniae). Pneumonia is a leading killer of young children in low-income countries; vaccinations from 2010 to the end of this year are estimated to have averted 500,000 deaths, according to the GAVI Alliance in Geneva, an international organization that facilitates vaccination.

Data from South Africa also point to another benefit of vaccination: stemming a rising tide of antibiotic resistance in the developing world. The country's introduction of a pneumococcal conjugate vaccine (PCV) in 2009 has not only reduced the overall incidence of invasive pneumococcal disease by about two-thirds in infants (the age group vaccinated) and in adults, but has also reduced penicillin-resistant infections in both groups.

This is the first time such benefits have been observed outside the developed world. 
The data should spur publichealth officials in low-income countries that have not yet adopted the vaccine to start using it, says Anne von Gottberg, a clinical microbiologist at the National Institute for Communicable Diseases in Johannesburg and leader of the study (see 'Protecting children'). Her group has reported the results at conferences but they have not yet been published. resistance is particularly stark in low-income countries, where overprescription and poor regulation combine with a higher disease burden and poor sanitation to increase the use of antimicrobial drugs. A recent survey by the World Health Organization found rates of resisthigh as $54 \%$. Reduced susceptibility of Streptococcus pneumoniae to penicillin was found worldwide, and topped $50 \%$ in some reports.

In North America, Europe and other well-off parts of the world, the introduction of pneumococcal vaccines in the early 2000s reduced cases of invasive pneumococcal disease by more than one-third in vaccinated children and in unvaccinated adults, who typically acquire infections from children. The vaccine also reduced the numbers of serious pneumococcal infections that were resistant to front-line antibiotics such as penicillin.

Between 1998 and 2008, a study in the United States found a $64 \%$ decrease in antibiotic-resistant pneumococci among children and a 45\% decrease among adults over 65 (L. M. Hampton et al. J. Infect. Dis. 205, 401-411; 2012). The different pneumococcal vaccines target a handful $(7,10$ or 13 , depending on the vaccine) of the more than 90 varieties (serotypes) of the pneumococcus, but those serotypes are among the most likely to develop antibiotic resistance. The result is a greater reduction in antibiotic-resistant strains in the population compared with sensitive strains.

Low-income countries began deploying pneumococcal vaccines around 2009, and
The problem of antibiotic ance in Klebsiella pneumoniae as cination against the pneumococcus, a leading cause of death in young ildren, is gaining ground in Africa.

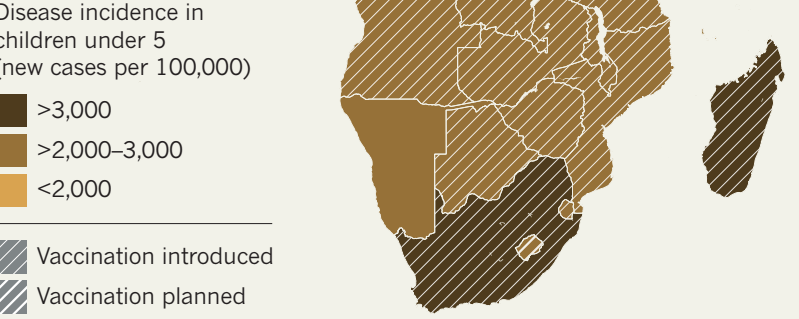

\section{PROTECTING CHILDREN}

tend to contract the disease along with children and older people.

Despite these differences, von Gottberg's team noticed a steep reduction in rates of invasive pneumococcal disease after vaccination began. By 2012, cases caused by the serotypes included in the vaccine had plummeted in both children and in middle-aged adults (by $89 \%$ and $57 \%$, respectively), and antibiotic-resistant infections with those serotypes were also down in the young (by $82 \%$ ) and across all age groups. The results suggest that the vaccine's effects in Western countries translated to children and adults in a sub-Saharan African country. It is notable, von Gottberg says, that the incidence of drug-resistant cases fell more than the incidence of drug-sensitive ones.

O'Brien says that the data suggest that other Sub-Saharan African countries should experience similar benefits after introducing the vaccine. Ongoing surveillance of the effects of the PCV10 vaccine roll-out in 2010 in a district in more than 40 of them are expected to administer the vaccine to infants by 2015. Many of these countries receive vaccines free or at a discount through financial support from the GAVI Alliance. "Getting those vaccines into low-income countries in a ten-year time span is an incredibly fast roll-out," says Katherine O'Brien, an epidemiologist at the Johns Hopkins Bloomberg School of Public Health in Baltimore, Maryland. She notes that most other vaccines have reached the world's poorest people much later after being introduced in developed countries.

South Africa, which is not eligible for GAVI support, funded its own pneumococcal vaccination programme in 2009 using the seven-strain PCV7, and von Gottberg's team tracked the effects. A different mix of pneumococcus serotypes circulates in South Africa from those in the Western countries for which the PCV7 vaccine was designed. And the types of people who contract pneumococcal disease also differ in South Africa, where HIV-infected adults, often mothers, severe pneumococcal infections (see go.nature. com/bxh6qp).

The reductions in antibiotic-resistant pneumococcal disease should lead to less use of antibiotics overall. A study in Finland found that the introduction of a different pneumococcal vaccine reduced antibiotic purchases by $8 \%$ (A. A. Palmu et al. Lancet Infect. Dis. 14, 205-212; 2014). Moreover, if doctors are confident that front-line antibiotics will routinely cure serious pneumococcal diseases, "they won't feel that impetus to have to go with a big-gun antibiotic", says O’Brien. More prudent use of antibiotics should forestall the development of drug resistance.

Von Gottberg hopes that her team's data will encourage countries that haven't yet signed up for the vaccine to follow Eritrea, Niger and Côte d'Ivoire. "To have another weapon in our armament to reduce antibiotic resistance is a very good story," she says. "It will convince governments."
Kenya has also recorded steep reductions in

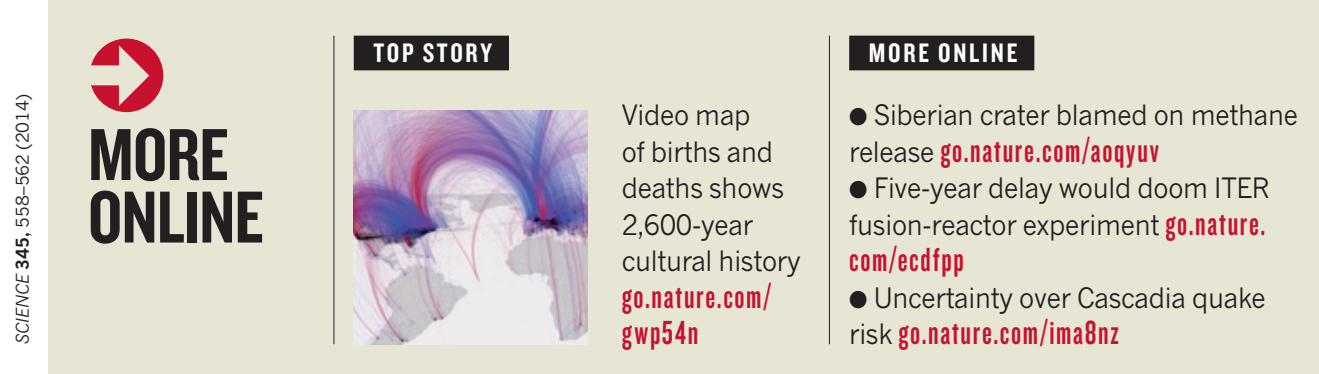

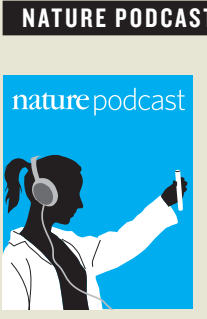

Straightening out nanotubes, chemistry's dream machine and an artist who fakes science nature.com/ nature/podcast 